\title{
Discriminative Power for Ensembles of Linear Decision Rules ${ }^{1}$
}

\author{
K. S. Kobylkin ${ }^{a, b}$ and M. Yu. Khachai ${ }^{a, b, c}$ \\ ${ }^{a}$ Institute of Mathematics and Mechanics, Ural Branch, Russian Academy of Sciences, \\ ul. Sof'i Kovalevskoi 16, Yekaterinburg, 620990 Russia \\ ${ }^{b}$ Ural Federal University, ul. Mira 19, Yekaterinburg, 620002 Russia \\ ${ }^{c}$ Omsk State Technical University, pr. Mira 11, Omsk, 644050 Russia \\ e-mail:kobylkin@imm.uran.ru,mkhachay@imm.uran.ru
}

\begin{abstract}
A novel class of ensembles of linear decision rules is introduced which includes majority votingbased ensembles as a particular case. Based on this general framework, new results are given that state the ability of a subclass to discriminate between two infinite subsets $A$ and $B$ in $R^{n}$, thus generalizing Mazurov's theorem for two finite sets.
\end{abstract}

Keywords: committee decision rule, separation of two sets.

DOI: $10.1134 /$ S1054661813030073

\section{INTRODUCTION}

The conventional formulation of the problem of machine learning for a class of majority voting-based decision rules [1] given samples $A$ and $B$ in $R^{n}$ from two classes is to find a positive integer $k$, vectors $d_{i} \in R^{n}$, thresholds $\alpha_{i} \in R$, and weights $w=\left[w_{i}\right]_{i=1}^{k} \in R_{+}^{k}$ such that, for a decision rule of the form

$$
f(\cdot)=H\left[\sum_{i=1}^{k} w_{i} H\left(\left(d_{i}, \cdot\right)-\alpha_{i}\right)-\frac{1}{2} \sum_{i=1}^{k} w_{i}\right]
$$

for any $a \in A$ the condition $f(a)=1$ is fulfilled (correspondingly, for any $b \in B$, the condition $f(b)=0$ is fulfilled), where

$$
H(x)=\left\{\begin{array}{l}
1, \quad x>0, \\
1 / 2, \quad x=0, \\
0, \quad x<0
\end{array}\right.
$$

is the Heaviside function and $(\cdot, \cdot)$ denotes the scalar product in $R^{n}$. Then decision rule (1.1) is called correct over sets $A$ and $B$. It is easy to see that rule $f$ gives 1 for vector $x \in R^{n}$ if

$$
\sum_{i: H\left(l_{i}(x)\right)=1} w_{i}>\sum_{i: H\left(l_{i}(x)\right)=0} w_{i}
$$

and yields 0 when the reverse strict inequality holds, where $l_{i}(\cdot)=\left(d_{i}, \cdot\right)-\alpha_{i}, i=1, \ldots, k$.

The notion of separating committee of hyperplanes (linear functions) is closely related to correct decision rules (1.1).

\footnotetext{
${ }^{1}$ The article was translated by the authors.
}

Received March 3, 2012
Definition 1.1 [1]. A set $K=\left\{\left(l_{i}, w_{i}\right)\right\}_{i=1}^{k}$, of pairs is called a committee of linear functions which discriminates between (separates) two subsets $A$ and $B$ of $R^{n}$ if inequalities

$$
\begin{aligned}
& \sum_{i: l_{i}(x)>0} w_{i}>q / 2, \quad \forall x \in A, \\
& \sum_{i: l_{i}(x)<0} w_{i}>q / 2, \quad \forall x \in B
\end{aligned}
$$

are satisfied where $w_{i} \in Z_{+}, i=1, \ldots, k, q=\sum_{i=1}^{k} w_{i}, Z_{+}$ denotes a set of positive integers. In addition, $q$ is called the number of elements of set $K$. Functions $l_{i}$ of the committee are called elements of $K$, whereas $w_{i}$ are called the weights (or multiplicities) of these elements.

The separating committee concept represents a simple generalization for a hyperplane that discriminates between two subsets. Furthermore, a committee $K=\left\{\left(l_{i}, w_{i}\right)\right\}_{i=1}^{k}$ of linear functions corresponds to a specific correct decision rule (1.1) such that the following inequalities hold true:

$$
\begin{aligned}
& \sum_{i: H\left(l_{i}(x)\right)=1} w_{i}>q / 2, \quad \forall x \in A, \\
& \sum_{i: H\left(l_{i}(x)\right)=0} w_{i}>q / 2, \quad \forall x \in B .
\end{aligned}
$$

It is obvious that (1.5) is stronger than the correctness condition. 


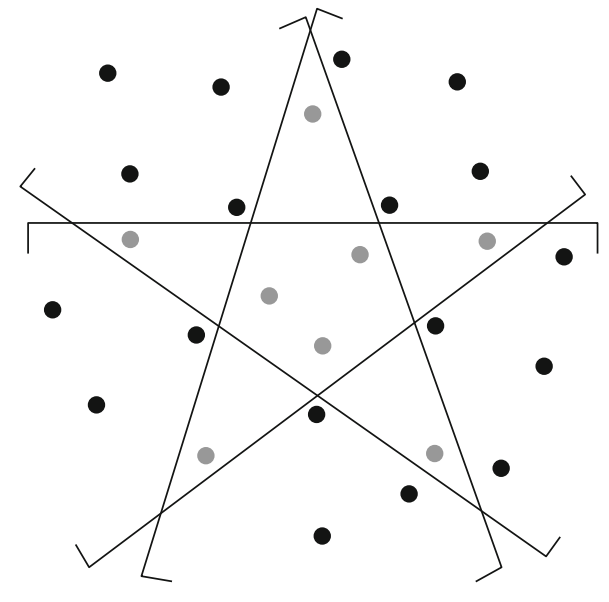

Figure. $(4,3,5)$-committee of five functions which discriminates between gray and black points. Its functions are depicted by their level sets $l(x)>0$. At every gray point at least four functions are positive, whereas for each black point at most three functions are nonnegative.

Theorem 1.1 [1]. A committee exists that separates two finite subsets $A$ and $B$ of $R^{n}$ iff $A \cap B=\varnothing$ where its number of elements does not exceed $|A \cup B|$.

Corollary [2]. The VC dimension for class of decision rules (1.1) is infinite.

It is also shown [2] that if $k \leq k_{0}$, then the $\mathrm{VC}$ dimension is $O\left(k_{0} n\right)$, where $k_{0}$ denotes some fixed positive integer.

The following problem is investigated.

Problem. What are the conditions for possibly infinite subsets $A$ and $B$ of $R^{n}$ under which a committee exists which discriminates between them?

Theorem 1.2 [3]. A committee exists separating two closed subsets $A$ and $B$ of $R^{n}$ iff $A \cap B=\varnothing$ and at least one of these subsets has a finite upper bound; as well one of the two has a finite lower bound.

Below, a separating $(s, t, q)$-committee concept is introduced (Def. 2.1), which covers separating committee notion (Def. 1.1) as a special case. Based on it a new technique is applied to give proofs for sufficient conditions under which two closed subsets $A$ and $B$ of $R^{n}$ with $A \cap B=\varnothing$ are separable by a committee.

\section{DEFINITION OF SEPARATING $(s, t, q)$-COMMITTEE}

Let $A$ and $B$ be subsets of $R^{n}, s$ and $t$ be positive integers with $s>t$.

Definition 2.1 A set $K=\left\{\left(l_{i}, w_{i}\right)\right\}_{i=1}^{k}$ of pairs is called an $(s, t, q)$-committee of linear functions that discriminates between two subsets $A$ and $B$ if the following inequalities hold true:

$$
\begin{aligned}
& \sum_{i: l_{i}(x)>0} w_{i} \geq s, \quad \forall x \in A, \\
& \sum_{i: l_{i}(x) \geq 0} w_{i} \leq t, \quad \forall x \in B,
\end{aligned}
$$

where $l_{i}(\cdot)=\left(d_{i}, \cdot\right)-\alpha_{i}, d_{i} \in R^{n}, \alpha_{i} \in R, w_{i} \in Z_{+}$, $i=1, \ldots, k, q=\sum_{i=1}^{k} w_{i}$.

An example of a $(4,3,5)$-committee is shown in the figure. Obviously, the $(s, t, q)$-committee defines some piecewise-linear surface which separates subsets $A$ and $B$. It also corresponds to a decision rule of the form

$$
f(\cdot)=H\left[\sum_{i=1}^{k} w_{i} H\left(l_{i}(\cdot)\right)-\frac{s+t}{2}\right],
$$

which is correct over sets $A$ and $B$. It is easy to show that the $(q, q-1, q)$-committee outlines the surface of a convex polyhedron $M$ containing set $A$ inside and set $B$ outside $R^{n / M}$. Furthermore, we obviously find that the $(s, t, q)$-committee becomes a committee for $s>q / 2>t$.

Suppose that set $A$ can be separated by a $(t+1, t$, $q$ )-committee from set $B$. Conversely, if $B$ is separable from $A$ by a surface of the same shape, then a $(q-t$, $q-1-t, q)$-committee exists that separates $A$ and $B$.

The notions of a committee and the $(s, t, q)$-committee are closely related. Consider a set $K=$ $\left\{\left(l_{i}, w_{i}\right)\right\}_{i=1}^{k}$ of pairs. Let us find out what the conditions are under which this set can be augmented to some committee that separates $A$ and $B$ by adding one of two functions $l_{T}(\cdot) \equiv(0, \cdot)+1$ or $l_{F}(\cdot) \equiv(0, \cdot)-1$ with some weight where 0 denotes zero vector in $R^{n}$.

Theorem 2.1. The set of pairs $K=\left\{\left(l_{i}, w_{i}\right)\right\}_{i=1}^{k}$ can be transformed to a committee that discriminates between sets $A$ and $B$ in $R^{n}$ by adding one of two functions $l_{T}(\cdot) \equiv(0, \cdot)+1$ or $l_{F}(\cdot) \equiv(0, \cdot)-1$ iff $K$ is a $(s, t, q)-$ committee that separates $A$ and $B$ for some positive integers $s$ and $t$ with $s>t$.

Proof. In the case when set $K$ transforms to a committee $\bar{K}$ by adding pair $\left(l_{T}, w_{k+1}\right)$, we have the following inequalities according to Def. 1.1:

$$
\begin{gathered}
\sum_{i \neq k+1, l_{i}(x)>0} w_{i}+w_{k+1}>p / 2, \quad x \in A, \\
\sum_{i \neq k+1, l_{i}(x)<0} w_{i}>p / 2, \quad x \in B,
\end{gathered}
$$


where $p=\sum_{i=1}^{k+1} w_{i}$. It can be assumed that $p$ is odd (a committee whose number of elements is even can be reduced to a committee by decrementing weight $w_{i}$ of one of its functions $l_{i}$ ). Set $s=\lceil p / 2\rceil-w_{k+1}$ and $t=\lfloor p / 2\rfloor-w_{k+1}$, where $\lfloor x\rfloor$ and $\lceil x\rceil$ denote rounding to the closest integer, which is less than or more than real $x$, respectively. In view of $w_{i} \in Z_{+}$for every $i=1, \ldots, k+1$ and keeping in mind that $p$ is odd, we obtain

$$
\begin{gathered}
\sum_{i \neq k+1, l_{i}(x)>0} w_{i} \geq s, \quad x \in A, \\
\sum_{i \neq k+1, l_{i}(x) \geq 0} w_{i} \leq t, \quad x \in B .
\end{gathered}
$$

If $K$ transforms to a committee $\bar{K}$ by adding pair $\left(l_{F}, w_{k+1}\right)$, the same path can be followed by setting $s=\lceil p / 2\rceil$ and $t=\lfloor p / 2\rfloor$.

$$
\text { Conversely, let } q=\sum_{i=1}^{k} w_{i} \text {. When } t<q / 2<s \text {, set } K \text { is }
$$

a committee that separates $A$ and $B$. Therefore, adding any two functions $l_{T}$ or $l_{F}$ with $w_{k+1}=0$ gives the committee $\bar{K}$. Now assume that $q / 2 \notin(t, s)$. Consider the case $q=2 h$ for some positive integer $h$. If $t \geq h$, we add the function $l_{F}$ to $K$ with $w_{k+1}=2(t-h)+1$. For the set $\bar{K}$ thus obtained we have $\sum_{i=1}^{k+1} w_{i}=2 t+1$. In view of $s>t$ and the inequalities

$$
\begin{gathered}
\sum_{i: l_{i}(x)>0} w_{i} \geq s \geq t+1, \quad x \in A, \\
\sum_{i: l_{i}(x) \geq 0} w_{i} \leq t, \quad x \in B
\end{gathered}
$$

we find that $\bar{K}$ is a committee. However, if $h>t$ then $h \geq s$. In this case let us add function $l_{T}$ to $K$ with $w_{k+1}=2(h-s)+1$. For the resulting set of pairs we

$$
\begin{aligned}
& \text { have } \sum_{i=1}^{k+1} w_{i}=2(2 h-s)+1 \text { and } \\
& \sum_{i: l_{i}(x)>0} w_{i} \geq s+2(h-s)+1=2 h-s+1, \quad x \in A, \\
& \sum_{i: l_{i}(x) \geq 0} w_{i} \leq t+2(h-s)+1 \leq 2 h-s, \quad x \in B,
\end{aligned}
$$

where summation is performed over all $i$ from 1 to $k+1$.

Now consider the case $q=2 h-1$. When $h>s$, we add function $l_{T}$ to $K$ with $w_{k+1}=2(h-s)$. For the set of pairs thus augmented we find that $\sum_{i=1}^{k+1} w_{i}=2(2 h-s)-1$ and $\sum_{i: l_{i}(x)>0} w_{i} \geq s+2(h-s)=2 h-s, \quad x \in A$,

$$
\sum_{i: l_{i}(x) \geq 0} w_{i} \leq t+2(h-s) \leq 2 h-s-1, \quad x \in B .
$$

In the case $h \leq s$, the inequality $h \leq t$ holds. Let us add function $l_{F}$ to $K$ with $w_{k+1}=2(t-h+1)$ and follow the same path. We have $\sum_{i=1}^{k+1} w_{i}=2 t+1$ and

$$
\begin{gathered}
\sum_{i: l_{i}(x)>0} w_{i} \geq s \geq t+1, \quad x \in A, \\
\sum_{i: l_{i}(x) \geq 0} w_{i} \leq t, \quad x \in B .
\end{gathered}
$$

The theorem is proved.

\section{SUFFICIENT CONDITIONS FOR EXISTENCE OF A COMMITTEE THAT DISCRIMINATES BETWEEN TWO INFINITE SUBSETS OF $R^{n}$}

Let us introduce some auxiliary constructions and notations. At first we describe union operation for two finite sets of pairs. Let $K$ and $L$ be two finite sets of pairs whose elements (first components of pairs, see Def. 1.1) belong to an arbitrary set $X$. Let us define the set of pairs $K^{\prime}$ in the following way. Each element of $K$ but not of $L$ is added to $K^{\prime}$ with the weight this element has for set $K$. Analogously, each element of $L$ not belonging to $K$ is added to $K^{\prime}$ with the same multiplicity as the one for $L$. Finally, each element which belongs to both $K$ and $L$ with weights $p_{1}$ and $p_{2}$, respectively, is included in $K^{\prime}$ with multiplicity $p_{1}+p_{2}$. Denote $K \cup L=: K^{\prime}$. For three sets $K_{1}, K_{2}$, and $K_{3}$ set $K_{1} \cup K_{2} \cup K_{3}:=\left(K_{1} \cup K_{2}\right) \cup K_{3}$. The union of four and more sets is defined in the same way.

In the sequel int $M$ denotes the interior of set $M$, whereas set $\mathrm{cl} M$ gives closure of $M$ and bd $M$ is its border. For simplicity we identify linear function $l$ with its level set (half-space) $P=\left\{x \in R^{n}: l(x)>0\right\}$, whereas separating committee of linear functions is the same as the set of pairs of the form (half-space, its weight). If $l(x) \equiv \alpha \in R$, the corresponding half-space $P$ is the empty set for $\alpha \leq 0$ and coincides with the whole space for $\alpha>0$. Also we say that open half-space $P$ votes for point $a \in A$ if $a \in P$ and votes for $b \in B$ if $b \notin \operatorname{cl} P$. 
If $P=\varnothing$, then $P$ votes for each point of $B$ (for set $B$ ) and against set $A$. In the opposite case, $P=R^{n}$ halfspace $P$ votes for set $A$ and against set $B$. It is easy to see that set of pairs $\left\{\left(l_{i}, w_{i}\right)\right\}_{i=1}^{k}$ is a committee that separates $A$ and $B$ iff for each point of $A \cup B$ a majority of half-spaces votes for in the corresponding set of pairs $\left\{\left(P_{i}, w_{i}\right)\right\}_{i=1}^{k}$, where $P_{i}=\left\{x \in R^{n}: l_{i}(x)>0\right\}, i=$ $1, \ldots, k$.

The following theorem generalizes theorem 1.1 in the case of infinite sets $A$ and $B$.

Theorem 3.1. If $A$ and $B$ are closed subsets of $R^{n}$ with $A \cap B=\varnothing$ such that one of them is bounded and has finite number of limiting points, then a committee exists that separates $A$ and $B$.

Proof. Let $A$ and $B$ be two closed subsets of $R^{n}$ with $A \cap B=\varnothing$ such that $A$ is bounded set having finite number of limiting points. It is easy to see that $A$ is countable or finite. As $A$ and $B$ are closed sets, $A$ is bounded and $A \cap B=\varnothing$, there exists such $\rho>0$ that any ball of radius $\rho$ centered at arbitrary point of $A$ does not contain points of $B$.

Let us give the following procedure. Let $A^{\prime}$ be an arbitrary finite subset of $A$ and $a \in A^{\prime}$ be some vertex of the convex hull conv $A^{\prime}$. Consider an arbitrary $n$ dimensional simplex $S$ containing convex hull conv $A^{\prime}$ which has point $a$ as one of its vertices. Get the $(n-1)$ dimensional face of $S$ which is opposite to $a$ and consider hyperplane $H$ parallel to that face which cuts off an $n$-dimensional simplex from $S$ that is contained in the ball centered at $a$ of radius $\rho$.

Simplex $S$ is the intersection of some set of $n+1$ closed half-spaces. Let $P$ be the half-space containing point $a$ and whose border is hyperplane $H$. Let us shift each of these $n+1$ half-spaces bounding $S$ in parallel and denote by $T$ the simplex thus obtained. We perform shifting in such a way that $S \subset \operatorname{int} T$ and $A \cap$ bd $T=\varnothing$. This last condition holds because $A$ is countable or finite. Moreover, we shift the half-space $P$ in parallel in such a way that the border of the shifted half-space starts to nip some $n$-dimensional simplex $T_{1}$ from simplex $T$ with $a \in \operatorname{int} T_{1}$ and $T_{1} \cap B=\varnothing$.

Simplices $T_{1}$ and $T$ have a common vertex, which we denote by $c_{1}$. Let $c_{2}, \ldots, c_{n+1}$ be the other vertices of $T$. For each $s=2, \ldots, n+1$ consider the cone $K_{s}$ bounded by $n$ different hyperplanes each of which passes through some $(n-1)$-dimensional face of $T$ containing point $c_{s}$ with $K_{s} \cap T=\left\{c_{s}\right\}$. Get the $(n-1)$ dimensional face of $T$ opposite to $c_{s}$. Using a hyperplane parallel to that face, cut off some simplex $T_{s}$ from simplex $T$ such that $T_{s} \cap A=\varnothing$. Such a construction is possible in view of $c_{s} \notin A$. The procedure is finished.

This procedure is used for set $A^{\prime}$ from which elements are deleted consecutively. In three stages we get a committee that separates $A$ and $B$.

STAGE 1. Let $A_{0}$ be the set of limiting points of bounded set A which is finite due to the theorem. Set
$t_{0}=\left|A_{0}\right|$ and $A^{\prime}=A_{0}$. Consider a system of simplices $T$, $T_{1}, \ldots, T_{n+1}$ provided by the procedure applied for set $A^{\prime}$ and for an arbitrary vertex $a_{1}$ of convex hull conv $A^{\prime}$. Let $V^{1}:=T$ and $V_{s}^{1}:=T_{s}, s=1, \ldots, n+1$. Setting $A^{\prime}:=$ $A^{\prime} \backslash\left\{a_{1}\right\}$ we apply the procedure for set $A^{\prime}$ and for an arbitrary vertex $a_{2}$ of convex hull conv $A^{\prime}$. As a result we have another system $T, T_{1}, \ldots, T_{n+1}$ of simplices. Let $V^{2}:=T$ and $V_{s}^{2}:=T_{s}, s=1, \ldots, n+1 \operatorname{set} A^{\prime}:=A^{\prime} \backslash\left\{a_{2}\right\}$. We continue repeating the procedure until set $A^{\prime}$ becomes empty. Obviously, sequence $a_{1}, \ldots, a_{t_{0}}$ thus obtained coincides with set $A_{0}$. For each $r=1, \ldots, t_{0}$ consider an open neighborhood $U\left(a_{r}\right)$ of point $a_{r}$ which is contained in $V_{1}^{r} \cap \bigcap_{i=1}^{r-1} V^{i}$ as a subset. Due to the boundedness of $A$, the set $A_{1}=A \backslash \bigcup_{r=1}^{t_{0}} U\left(a_{r}\right)$ is finite.

In the case $A_{0}=\varnothing$, we set $A^{\prime}:=A_{1}=A$ and $p:=1$ going to stage 2 . Otherwise, for $A_{0} \neq \varnothing$ we apply the following simplex generation process. Set $p:=1$ and $A^{\prime}=A_{1} \cup \operatorname{ver} V^{1}$, where $\operatorname{ver} V^{1}$ is the vertex set for simplex $V^{\prime}$. When convex hull conv $A^{\prime}$ does not coincide with $V^{1}$, some point $a \in A_{1}$ will be a vertex of the hull. Obtain simplices $T, T_{1}, \ldots, T_{n+1}$ applying the procedure for the set $A^{\prime}$ and the point $a$. Set $a_{1}^{\prime}:=a, T^{1}:=$ $T, T_{s}^{1}:=T_{s}$, where $s=1, \ldots, n+1$. Then set $A^{\prime}:=$ $A^{\prime} \backslash\{a\}$ and $p:=2$. If the convex hull conv $A^{\prime}$ still does not equal to the simplex $V^{1}$ consider an arbitrary point $a \in A_{1}$ being a vertex of the hull. By applying the procedure for the set $A^{\prime}$ and the point $a$ we get another simplex series $T, T_{1}, \ldots, T_{n+1}$. Again set $a_{2}^{\prime}:=a, T^{2}:=$ $T, T_{s}^{2}:=T_{s}, s=1, \ldots, n+1$. Let $A^{\prime}:=A^{\prime} \backslash\{a\}$ and $p:=3$. While $\operatorname{conv} A^{\prime} \neq V^{1}$ we continue going in the same way.

Due to the fact that $A_{1} \backslash V^{1}$ is finite, we finally arrive at the case $\operatorname{conv} A^{\prime}=V^{1}$ for some $p=p_{1}$. Set $a_{p_{1}}^{\prime}:=a_{1}$, $T^{p_{1}}:=V^{1}, T_{s}^{p_{1}}:=V_{s}^{1}$, where $s=1, \ldots, n+1$. Let $p:=$ $p_{1}+1$. We now follow the same way of simplex generation for $r=2$. Specifically, let $A^{\prime}:=\left(A^{\prime} \backslash\right.$ ver $\left.V^{r-1}\right) \cup$ ver $V^{r}$. If the convex hull conv $A^{\prime}$ does not coincide with $V^{r}$, let us get a vertex $a$ of the hull that is contained in $A_{1}$. By applying the procedure for set $A^{\prime}$ and point $a$, we obtain a system $T, T_{1}, \ldots, T_{n+1}$ of simplices. Then we set $a_{p}^{\prime}:=a, T^{p}:=T, T_{s}^{p}:=T_{s}, s=1, \ldots, n+1$ and let $A^{\prime}:=A^{\prime} \backslash\{a\}$ and $p:=p+1$. While conv $A^{\prime} \neq V^{r}$ we continue repeating this process. Again we come to the case where $\operatorname{conv} A^{\prime}=V^{r}$ for some $p=p_{r}$. Then let $a_{p_{r}}^{\prime}:=a_{r}$, $T^{p_{r}}:=V^{r}, T_{s}^{p_{r}}:=V_{s}^{r}, s=1, \ldots, n+1$ and set $p:=p_{r+1}$. 
The whole process works for $r=3,4, \ldots, t_{0}$. At the end we delete from $A^{\prime}$ all vertices of simplex $V^{t_{0}}$. It is easy to see that $A^{\prime}=A_{1} \cap \bigcap_{i=1}^{t_{0}} V^{r}$.

STAGE 2 . In the case $A^{\prime} \neq \varnothing$ we apply the procedure for set $A^{\prime}$ and for an arbitrary vertex of its convex hull. Having provided the system of simplices $T$, $T_{1}, \ldots, T_{n+1}$, we set $a_{p}^{\prime}:=a, T^{p}:=T, T_{s}^{p}:=T_{s}, s=$ $1, \ldots, n+1$. Also let $A^{\prime}:=A^{\prime} \backslash\{a\}$ and $p:=p+1$. Finally, while set $A^{\prime}$ is nonempty we continue doing the same.

STAGE 3. At the end of stage 2 , we have two families $\left\{T^{k}\right\}$ and $\left\{T_{s}^{k}\right\}$ of simplices where $s=1, \ldots, n+1$ and $k=1, \ldots, t, t=\left|A_{0}\right|+\left|A_{1}\right|$. For each $k, 1 \leq k \leq t$, define a series of open half-spaces $\left\{P_{i}^{k}\right\}_{i=1}^{2 n+2}$ by the conditions $\operatorname{int} T^{k}=\bigcap_{i=1}^{n+1} P_{i}^{k} \operatorname{int} T_{1}^{k}=\bigcap_{i=2}^{n+2} P_{i}^{k}$ and $\operatorname{int} T_{s}^{k}=Q_{n+1+s}^{k} \cap \bigcap_{i=1, i \neq s}^{n+1} Q_{i}^{k}$, where $2 \leq s \leq n+1$ and $Q_{i}^{k}$ is the open half-space distinct from $P_{i}^{k}$, which has a common boundary with $P_{i}^{k}$. Equip set $L_{k}$ of pairs with these half-spaces taken with some multiplicities. Each of the half-spaces $\left\{P_{i}^{k}\right\}_{i=1}^{n+1}$ will have weight $n \cdot n^{2(t-k)}$, whereas each of the half-spaces $\left\{P_{i}^{k}\right\}_{i=n+2}^{2 n+2}$, the weight $n^{2(t-k)}$. Let $K_{0}=\left\{\left(P_{0}, w_{0}\right)\right\}$, where $P_{0}=\left\{x \in R^{n}:(0, x)>1\right\}$ and $w_{0}=n^{2 t}$. Set $K=$ $K_{0} \cup L_{1} \cup \ldots \cup L_{t}$, where symbol $\cup$ denotes union operation. Stage 3 is finished.

Let us show that $K$ is a committee. Consider the case where $n \neq 1$. Since the number of elements for the set $L_{k}$ is equal to $(n+1)^{2} n^{2(t-k)}, k=1, \ldots, t$, the number of elements for $K$ is equal to

$$
\begin{gathered}
(n+1)^{2}\left(n^{2(t-1)}+n^{2(t-2)}+\ldots+1\right)+n^{2 t} \\
=2 n^{2 t}-1+\frac{2}{n-1}\left(n^{2 t}-1\right) .
\end{gathered}
$$

Let us count the number of elements of $L_{k}, k=1, \ldots, t$ which vote for some $a \in A$. Due to the procedure, two situations are possible for each point $a \in A$ : $a$ is in the interior of simplex $T^{k}$; otherwise it lies in its open exterior. If $a \in \operatorname{int} T^{k}$, the half-spaces $\left\{P_{i}^{k}\right\}_{i=1}^{n+1}$ vote for point $a$ by their multiplicities $n \cdot n^{2(t-k)}$, totaling $(n+1) n \cdot n^{2(t-k)}$ votes. Moreover, if $a \in \operatorname{int} T_{1}^{k}$, halfspace $P_{n+2}^{k}$ votes for $a$ by its weight $n^{2(t-k)}$ and each of the half-spaces $\left\{P_{i}^{k}\right\}_{i=1}^{n+1}$, by their multiplicities $n \cdot n^{2(t-k)}$, which amounts to $((n+1) n+1) n^{2(t-k)}$ votes. Two cases are possible for $a \notin T^{k}$. The first case takes place when $a \in P_{i_{1}}^{k} \cap P_{i_{2}}^{k}$ for some $i_{1}$ and $i_{2}, 1 \leq$ $i_{1}, i_{2} \leq n+1$. The second case is where $a \in$ $\bigcap^{n+1} \operatorname{cl} Q_{i}^{k}$, for some $i_{0}, 1 \leq i_{0} \leq n+1$. If the first case $i=1, i \neq i_{0}$

holds, there are at least $2 n \cdot n^{2(t-k)}$ votes for point $a$ in set $L_{k}$. In the second case, in view of $T_{s}^{k} \cap A=\varnothing$ for every $s=2, \ldots, n+1$ and taking inclusion $P_{n+2}^{k} \supset$ $\bigcap \operatorname{n+1} \mathrm{cl} Q_{i}^{k}$ into account, we find that half-space $P_{i_{0}}^{k}$ $i=2$

votes for point $a$ a by its weight $n \cdot n^{2(t-k)}$, while halfspace $P_{n+1+i_{0}}^{k}$ does the same by its multiplicity $n^{2(t-k)}$, which totals $(n+1) n^{2(t-k)}$ votes in $L_{k}$.

Let us count the number of elements of $K$ which vote for some point $a \in A$. Obviously, the finite sequence $a_{1}^{\prime}, \ldots, a_{t}^{\prime}$ of distinct points that forms at stages 1 and 2 coincides with set $A_{0} \cup A_{1}$. In view of equality $A=A_{1} \cup \bigcup_{a^{\prime} \in A_{0}}\left(U\left(a^{\prime}\right) \cap A\right)$, we have either $a=$ $a_{p}^{\prime}$ for some $p, 1 \leq p \leq t$ or $a \in U\left(a^{0}\right)$, where $a^{0} \in A_{0}$. Let us count the number of votes for the first case. Due to the generation procedure for simplices $\left\{T^{k}\right\}$ and $\left\{T_{s}^{k}\right\}$, point $a$ lies inside the intersection $T_{1}^{p} \cap \bigcap_{1 \leq k<p} T^{k}$. Consequently, for $p \neq 1$ and $p \neq t$, the number of elements of $K$ which vote for $a$ is greater than or equal to

$$
\begin{gathered}
(n+1) n\left(n^{2(t-1)}+\ldots+n^{2(t-p+1)}\right) \\
+((n+1) n+1) n^{2(t-p)} \\
+(n+1)\left(n^{2(t-p-1)}+\ldots+1\right)=n^{2 t}-n^{2(t-p+1)} \\
+\frac{1}{n-1}\left(n^{2 t}-n^{2(t-p+1)}\right)+n^{2(t-p+1)} \\
+\frac{1}{n-1} n^{2(t-p+1)}-\frac{1}{n-1} n^{2(t-p)} \\
+\frac{1}{n-1} n^{2(t-p)}-\frac{1}{n-1}=n^{2 t}+\frac{1}{n-1}\left(n^{2 t}-1\right) .
\end{gathered}
$$

For $p=1$, this number is greater than or equal to

$$
\begin{gathered}
((n+1) n+1) n^{2(t-1)}+(n+1)\left(n^{2(t-2)}+\ldots+1\right) \\
=n^{2 t}+\frac{1}{n-1}\left(n^{2 t}-1\right) .
\end{gathered}
$$

Analogously, for $p=t$ the number is at least as great as

$$
\begin{gathered}
(n+1) n\left(n^{2(t-1)}+\ldots+n^{2}\right)+((n+1) n+1) \\
=n^{2 t}+\frac{1}{n-1}\left(n^{2 t}-1\right) .
\end{gathered}
$$


Now consider the case $a \in U\left(a^{0}\right)$, where $a^{0}$ is some point of set $A_{0}$. Since $a^{0}=a_{p}^{\prime}$ for some $p, 1 \leq p \leq t$, point $a$ lies inside intersection $T_{1}^{p} \cap \bigcap_{1<k<p} T^{k}$ by the construction of neighborhood $U\left(a^{0}\right)$ and series of simplices $\left\{T^{k}\right\}$ and $\left\{T_{s}^{k}\right\}$. As a consequence, there are at least $n^{2 t}+\frac{1}{n-1}\left(n^{2 t}-1\right)$ votes in $K$ for point $a$.

For every point $b \in B$, let us now count the number of elements of $L_{k}, k=1, \ldots, t$, which vote for $b$. If $b \in T^{k}$, we have $b \notin T_{1}^{k}$ according to the construction of simplex $T_{1}^{k}$. For this point, each of the half-spaces $\left\{P_{i}^{k}\right\}_{i=n+2}^{2 n+2}$ votes by its weight $n^{2(t-k)}$, which overall amounts to $(n+1) n^{2(t-k)}$ votes. In the case of $b \notin T^{k}$, some half-space $P_{i_{0}}^{k}, 1 \leq i_{0 \leq n+1}$, votes for $b$ by its multiplicity $n \cdot n^{2(t-k)}$ and so does half-space $P_{n+1+i_{0}}^{k}$ by its weight $n^{2(t-k)}$, whose boundary is parallel to that for $P_{i_{0}}^{k}$. It sums up to give at least $(n+1) n^{2(t-k)}$ votes for $b$ in $L_{k}$. Due to the fact that $P_{0}$ votes for the $B$ by its multiplicity $n^{2 t}$, the number of elements of $K$ which vote for point $b$ is at least as much as $(n+1)\left(n^{2(t-1)}+\right.$ $\ldots+1)+n^{2 t}=n^{2 t}+\frac{1}{n-1}\left(n^{2 t}-1\right)$, so $K$ is a committee.

For $n=1$ it is easy to count that the number of elements of $K$ is equal to $4 t+1$, while the number of elements voting for an arbitrary point of $A \cup B$ is greater than or equal to $2 t+1$. The theorem is proved.

Remark. Using more general notion of an $(s, t, q)$ committee yields a simpler proof for the case where set $A$ is finite.

The boundedness condition imposed on one of two sets being separated is essential.

Example. There is no committee which separates sets $A=\{2 k\}_{k=1}^{\infty}$ and $B=\{2 k-1\}_{k=1}^{\infty}$.

In contrast, if such a committee exists, there is a linear function in $K$ having a positive weight that takes positive value for $2 k$ and gives a negative value for $2 k-$ 1 , where $k$ is an arbitrary positive integer. Due to the fact that these functions are different for distinct $k$ and using the finiteness of the committee, we find that a committee that separates these two sets does not exist.

Let us give the classical result on the external approximation of solid convex compact by a convex polyhedron to arbitrary accuracy (see, e.g., [5]). We call

$$
\begin{gathered}
\delta\left(C_{1}, C_{2}\right)=\max \left[\sup \left\{\rho\left(x, C_{2}\right): x \in C_{1}\right\},\right. \\
\left.\sup \left\{\rho\left(x, C_{1}\right): x_{2} \in C_{2}\right\}\right]
\end{gathered}
$$

the Hausdorff metric between two convex compact subsets $C_{1}$ and $C_{2}$ of $R^{n}$ having a nonempty interior (which are called convex bodies), where $\rho(x, C):=$ $\inf \{|x-y|: y \in C\}$ and $|\cdot|$ denotes the Euclidean norm. For a given convex body $C$, we consider convex hulls of finite sets with at most $m$ faces (facets having the maximal dimension), which contain set $C$ and touch its boundary where $m$ is given positive integer. We denote by $P_{m}^{c}(C)$ the set of all convex hulls of this form and set $\delta\left(C, P_{m}^{c}(C)\right)=\inf \left\{\delta(C, D): D \in P_{m}^{c}(C)\right\}$.

Theorem 3.2 [5]. The following equality holds true: $\lim _{m \rightarrow \infty} \delta\left(C, P_{m}^{c}(C)\right)=0$.

The following theorem is closely related to the classical result on the separability of two convex sets by a hyperplane.

Theorem 3.3. If $A$ and $B$ are closed subsets of $R^{n}$ with an empty intersection where $A$ is a convex body, then $A$ and $B$ are separable by a committee.

Proof. Since $A$ is compact, $B$ is closed and $A \cap B=$ $\varnothing$ we have

$$
\rho(A, B):=\inf \{|x-y|: x \in A, y \in B\}=\varepsilon_{0}>0 .
$$

Set $A(\varepsilon)=\bigcup_{x \in A} O_{x}(\varepsilon)$ is a compact body, where $O_{x}(\varepsilon)$ is an $n$-dimensional ball centered at point $x \in R^{n}$ of radius $\varepsilon$. Set $A_{0}=A\left(\varepsilon_{0} / 4\right)$. Then $\rho\left(A_{0}, B\right)>\varepsilon_{0} / 2$. Choose a positive integer $m$ large enough to have $\delta\left(A_{0}\right.$, $\left.P_{m}^{c}\left(A_{0}\right)\right)<\varepsilon_{0} / 4$. Therefore, there exists $M \in P_{m}^{c}\left(A_{0}\right)$ such that $A_{0} \subseteq M$ and $M \cap B=\varnothing$ so that $A \subseteq \operatorname{int} M$. Because $M$ coincides with the intersection of a finite number (say, $t \leq m$ ) of closed half-spaces, there exists a $(t, t-1, t)$-committee that separates $A$ and $B$. Then, according to theorem 2.1, there exists a committee that separates them. The theorem is proved.

\section{CONCLUSIONS}

A new concept of the $(s, t, q)$-committee decision rule is introduced which includes the committee decision rule as a special case. A series of sufficient conditions for two subsets $A$ and $B$ of $R^{n}$ is considered under which there exists a correct decision rule where $A$ and $B$ are not finite in general. These results generalize Vl.D.Mazurov's famous criterion on the separability of two finite subsets of $R^{n}$ having an empty intersection. 


\section{ACKNOWLEDGMENTS}

This work was supported by the Ural Branch of the Russian Academy of Sceinces (project nos. 12-P-11016 and 12-C-1-1017/1), by the Russian Foundation for Basic Research (project nos. 13-01-00210 and 13-07-00181), and by a 2013 grant nos. 10-01-00273, 10-07-00134 from the Institute of Mathematics and Mechanics, Ural Branch, Russian Academy of Sciences.

\section{REFERENCES}

1. Vl. D. Mazurov, Committee Method in Optimization and Classification Problems (Science, Moscow, 1990) [in Russian].



Konstantin S. Kobylkin. Born 1977. Received master's (Math.) degree at Ural State University in 2001. Received canditate's degree with specialization "Discrete Mathematics and Mathematical Cybernetics" 2005. Since 2001 working for Institute Of Mathematics And Mechanics, Ural Branch, Russian Academy of Sciences. Current position: senior researcher. Since 2004 working for Ural Federal University. Scientific interests: data mining, pattern recognition theory, signal processing. Author of ten publications. Member of IAPR.
2. M. Yu. Khachai, "Sufficient training sample length for committee decision rule," Iskusstv. Intellekt, No. 2, 219-223 (2000).

3. A. I. Krivonogov, "Some grounding problems for committee algorithm," in Classification and Optimization in Control Problems (Ufa Sci. Center of RAS, Sverdlovsk, 1981), pp. 39-51 [in Russian].

4. K. S. Kobylkin, "Committee existence problems for linear inequalities," Available from VINITI no. 430V2005 (Yekaterinburg, 2005) [in Russian].

5. G. K. Kamenev, Optimal Adaptive Methods of Polyhedral Approximation for Convex Bodies (Dorodnicyn Computing Centre of RAS, Moscow, 2007) [in Russian].



Mikhail Yu. Khachai. Born 1970 in Krasnotur'insk, Sverdlovsk oblast. Graduated from Ural State University in 1993. Received candidates degree at the Institute of Mathematics and Mechanics in 1996 and doctoral degree at Dorodnicyn Computing Centre in 2005. Joined Institute of Mathematics and Mechanics, Ural Branch, Russian Academy of Sciences in 1994. Since 2007 head of mathematical programming department at this institute. Professor of Ural Federal University and visiting professor at Omsk State Technical University. Scientific interests: learning theory, combinatorial optimization, and computational complexity. Author of over 100 papers among them 15 in PRIA. Member of IAPR. 